\title{
Precision community: a mixed methods study to identify determinants of adoption and implementation of targeted cancer therapy in community oncology
}

Jenna Ball', Jeffrey Thompson', Elizabeth Wulff-Burchfield', Edward Ellerbeck', Kim Kimminau', Joanna Veazey Brooks' ${ }^{1}$, Shariska Petersen ${ }^{1}$, Duncan Rotich¹, Anita Y. Kinney ${ }^{2}$ and Shellie D. Ellis ${ }^{1 *}$ (D)

\begin{abstract}
Background: Precision medicine has enormous potential to improve cancer outcomes. Over one third of the 1.5 million Americans diagnosed with cancer each year have genetic mutations that could be targeted with an FDAapproved drug to treat their disease more effectively. However, the current uptake of targeted cancer therapy in clinical practice is suboptimal. Tumor testing is not widely used, and treatments based on molecular and genomic profiling are often not prescribed when indicated. Challenges with the uptake of precision medicine may disproportionately impact cancer patients in rural communities and other underserved populations. The objective of this study is to identify the determinants of adoption and implementation of precision cancer therapy to design an implementation strategy for community oncology practices, including those in rural areas.
\end{abstract}

Methods: This study is an explanatory sequential mixed methods study to identify factors associated with the use of targeted cancer therapy. Levels of targeted therapy use will be ascertained by secondary analysis of medical records to identify concordance with 18 national guideline recommendations for use of precision medicine in the treatment of breast, colorectal, lung, and melanoma skin cancer. Concurrently, facilitators and barriers associated with the use of precision cancer therapy will be elicited from interviews with up to a total of 40 oncologists, administrators, pathology, and pharmacy staff across the participating sites. Qualitative analysis will be a template analysis based on the Theoretical Domains Framework. Quantitative data aggregated at the practice level will be used to rank oncology practices' adherence to targeted cancer therapy guidelines. Determinants will be compared among high and low users to isolate factors likely to facilitate targeted therapy use. The study will be conducted in eight community oncology practices, with an estimated 4121 targeted therapy treatment decision-making opportunities over a 3-year period.

Discussion: Despite unprecedented investment in precision medicine, translation into practice is suboptimal. Our study will identify factors associated with the uptake of precision medicine in community settings. These findings will inform future interventions to increase equitable uptake of evidence-based targeted cancer treatment.

Keywords: Precision medicine, Implementation science, Cancer care delivery, Guideline adherence, Community practice

\footnotetext{
* Correspondence: Sellis4@kumc.edu

'Department of Population Health, University of Kansas School of Medicine

3901 Rainbow Boulevard, Kansas City, KS 66160, USA

Full list of author information is available at the end of the article
}

C C The Author(s). 2020 Open Access This article is licensed under a Creative Commons Attribution 4.0 International License, which permits use, sharing, adaptation, distribution and reproduction in any medium or format, as long as you give appropriate credit to the original author(s) and the source, provide a link to the Creative Commons licence, and indicate if changes were made. The images or other third party material in this article are included in the article's Creative Commons licence, unless indicated otherwise in a credit line to the material. If material is not included in the article's Creative Commons licence and your intended use is not permitted by statutory regulation or exceeds the permitted use, you will need to obtain permission directly from the copyright holder. To view a copy of this licence, visit http://creativecommons.org/licenses/by/4.0/. The Creative Commons Public Domain Dedication waiver (http://creativecommons.org/publicdomain/zero/1.0/) applies to the data made available in this article, unless otherwise stated in a credit line to the data. 


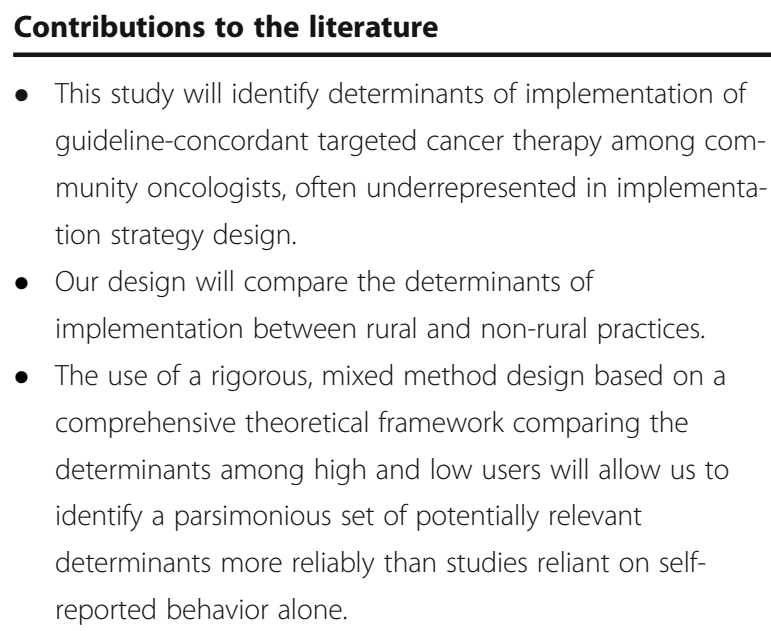

- This study will identify determinants of implementation of guideline-concordant targeted cancer therapy among community oncologists, often underrepresented in implementation strategy design.

- Our design will compare the determinants of implementation between rural and non-rural practices.

- The use of a rigorous, mixed method design based on a comprehensive theoretical framework comparing the determinants among high and low users will allow us to identify a parsimonious set of potentially relevant determinants more reliably than studies reliant on selfreported behavior alone.

\section{Background}

An estimated 1.5 million Americans are diagnosed with cancer each year [1]. Among the five most prevalent cancers (i.e., lung, breast, colorectal prostate, and melanoma), 38\% of patients' tumors have a genetic mutation that can be targeted with a therapy shown to be superior to standard treatment [2, 3]. Targeted therapies (TTs) can result in better treatment outcomes, including delay in tumor progression, longer survival [4], more qualityadjusted life-years [4], avoidance of ineffective treatment [5], and, ultimately, lower treatment costs $[4,6]$.

Across all cancer types, non-metropolitan rural areas have lower age-adjusted cancer incidence rates than metropolitan areas. However, non-metropolitan rural residents experience higher death rates than metropolitan area residents and have not benefited from recent reductions in cancer mortality [7]. Instead, rural/urban disparities are widening [7]. These differences in cancer mortality likely reflect disparities in the quality of and access to health care. The largest study to date comparing rural and urban patients' cancer outcomes demonstrated that when rural cancer patients are provided the same quality of care as urban patients, rural cancer patients have similar survival [8]. In particular, rural/urban disparities have been demonstrated in the delivery of targeted therapy [9] and associated with poorer survival [10]. Ensuring rural cancer patients' tumors are tested and appropriately treated with the 94 FDA-approved pharmacogenomic drugs [11] currently available could close the widening gap in cancer mortality.

Despite numerous benefits of TTs, the uptake of precision medicine in clinical practice is poor [10, 12, 13]. Among recently developed TTs, only $20 \%$ of stage IV non-small cell lung cancer patients in the USA receive guideline-recommended EGFR testing, and less than half of those eligible for EGFR inhibitors receive them [14]. Even among well-established TTs, testing and treatment are suboptimal. Only $23 \%$ of eligible stage IV colorectal cancer patients receive KRAS testing [10], and fewer than $50 \%$ of eligible breast cancer patients receive molecular testing to assess the need for adjuvant chemotherapy [13]. Furthermore, only half of white elderly women and only $40 \%$ of black elderly women with non-metastatic HER2positive breast cancer receive appropriate monoclonal antibody therapy [15]. Thus, there is ample opportunity to improve the delivery of precision medicine.

Research to identify barriers to targeted therapy is emerging, but incomplete. Previous work has identified deficits in infrastructure $[16,17]$, knowledge and skills $[17,18]$, access to tests and treatments [12,18], and time for coordination of testing, treatment, and follow-up [19-21] as factors associated with poor uptake of TTs. However, many domains relevant to the adoption and implementation of novel, guideline-based TTs (e.g., BRAF testing in metastatic melanoma) have not been assessed. Individual physician-level factors have been demonstrated to be influential in test ordering and prescribing decisions in other contexts [22, 23]. However, little is known about health care providers' influences around using targeted therapy $[24,25]$. Specific determinants, such as oncologists' peer support for use of TTs, alignment of the steps required to deliver it with oncologists' professional role and identity, beliefs about their capabilities to deliver it, and beliefs about positive and negative consequences of using TTs, have not been studied. Because of the more decentralized and hierarchical structure of many community specialty practices, it may be necessary to intervene upon these motivational factors to influence not only the initial decision to adopt TTs, but also in overcoming challenges to sustained use [12, 26, 27].

Underappreciation of the care delivery context may be one of the largest barriers to increasing adoption and implementation of TTs. State-of the-art TTs have been developed by scientists in resource-intensive institutions with high motivation and incentive to use them [28-36]. Yet, $79 \%$ of oncologists practice in non-academic settings [37] which are typically smaller, hematology/oncology practices with far fewer organizational resources available to implement TTs. Thus, the underlying determinants of adoption and implementation may be different in community practice than in academic practice. While physicians may be adopters of new innovations, especially in community practice, ensuring that it is fully implemented requires not only physician adoption, but also staff and administrative support. Our previous works suggest community cancer specialists may have unique practice organization, and practice pressures than those practicing in academic medical centers which may impact the effectiveness of proposed implementation strategies [38]. Further, recency of some targeted therapy availability, degree of service line organization, institutional capacity, and limited specialization relative 
to academic medicine may alter the balance of targeted therapy across cancer types, engendering variation in use and affecting underlying determinants of implementation within community oncology practice. To be effective, implementation strategies should address the targeted needs of providers and practices facing each of these particular challenges. However, little is known about community oncology providers' perspectives on TTs and variation in approach across community practices [36, 39, 40].

Our long-term objective is to design strategies to increase the adoption and implementation of targeted therapy consistent with the national guidelines among community oncologists. To accomplish this, we seek to explore context-specific organizational and provider characteristics associated with appropriate use, comparing determinants of adoption and implementation between high and low users, to better understand factors supportive of TT use in the spectrum of rural and nonrural community oncology practice.

\section{Study aims}

Aim 1: Engage community oncologists to assess the variation in targeted therapy use to identify facilitators and barriers to precision medicine adoption

We will conduct a secondary analysis of medical record data to identify appropriate use of targeted cancer treatment guidelines and assess provider and practice characteristics associated with guideline-concordant use of TTs. We hypothesize community oncologists will exhibit greater within-site variation in the use of TTs than between-site variation, that patient rurality will be a significant predictor of TT guideline use, and that practice size will be a significant organizational predictor of use.

\section{Aim 2: Isolate barriers to successful precision medicine implementation in community oncology practice}

We will conduct linked, semi-structured qualitative interviews of physicians, staff, and administrators involved in precision medicine implementation. Using the Theoretical Domains Framework (TDF), we will identify constructs key to implementation success and further describe the strength, frequency, and type of implementation strategies used by successful organizations. We anticipate that barriers and implementation strategies will be similar among adopters and non-adopters but that strength and frequency may differentiate implementation success.

\section{Methods}

\section{Overall design}

Together, the studies proposed in aims 1 and 2 represent an explanatory sequential mixed methods study design [41]. The mixed methods approach extends throughout data collection and interpretation by linking the data through the sampling frame and merging the quantitative and qualitative data for analysis [41]. Independently, aim 1 is a retrospective, cross-sectional analysis of extant medical records to identify organizational characteristics associated with appropriate use of TTs. Aim 2 is a qualitative comparative study of high and low TT users to identify behavioral determinants of TT implementation. The study design was aligned with the Standards for Reporting Implementation Studies (StaRI) statement [42, 43] and the Standards for Reporting Qualitative Research (SRQR) to ensure appropriate reporting upon publication [44].

\section{Sample and selection}

The sample consists of non-academic group practice organizations delivering care to adult cancer patients for whom TTs may be beneficial and comprised of oncology practices and health systems in which they operate. Individual physicians, staff, and administrators will be the target of both treatment observations and interviews aggregated to the service line and organizational level. Oncology practices in 13 states are included in the sampling frame (AR, CO, IA, IN, KS, MN, MO, NE, NM, OK, TX, WI, UT). We chose these states based on our existing clinical research networks and the potential to build capacity to support future methodologically robust implementation studies. Together, these states represent populations with higher than average rurality [45], who suffer cancer morbidity disproportionately [46], are understudied with regard to cancer care delivery [47], and are largely underrepresented in research $[48,49]$.

Using methods originated by the American Society of Clinical Oncology (ASCO) [37], we will create a list of non-academic oncology practices in the 13-state region from which to recruit participants. The list was derived from the Center for Medicare and Medicaid Services' Physician Compare database, a national, publicly available data set of all physicians billing Medicare. As the goal of the study is to differentiate high vs low TT adherence, we will structure the sampling frame to represent practices with characteristics associated with TT use to increase the probability that our selected sample includes both high and low TT users. We will partition the sampling frame based on measurable characteristics known to be associated with precision medicine use: insurance coverage climate (defined as states who have/ have not instituted Medicaid expansion, as similarity to existing coverage is one of the primary factors associated with expansion), rurality (defined as metropolitan vs non-metropolitan), and cancer volume (defined as practice size $2-5$ physicians or $6+$ physicians) as commonly defined by ASCO [37] (Fig. 1). 


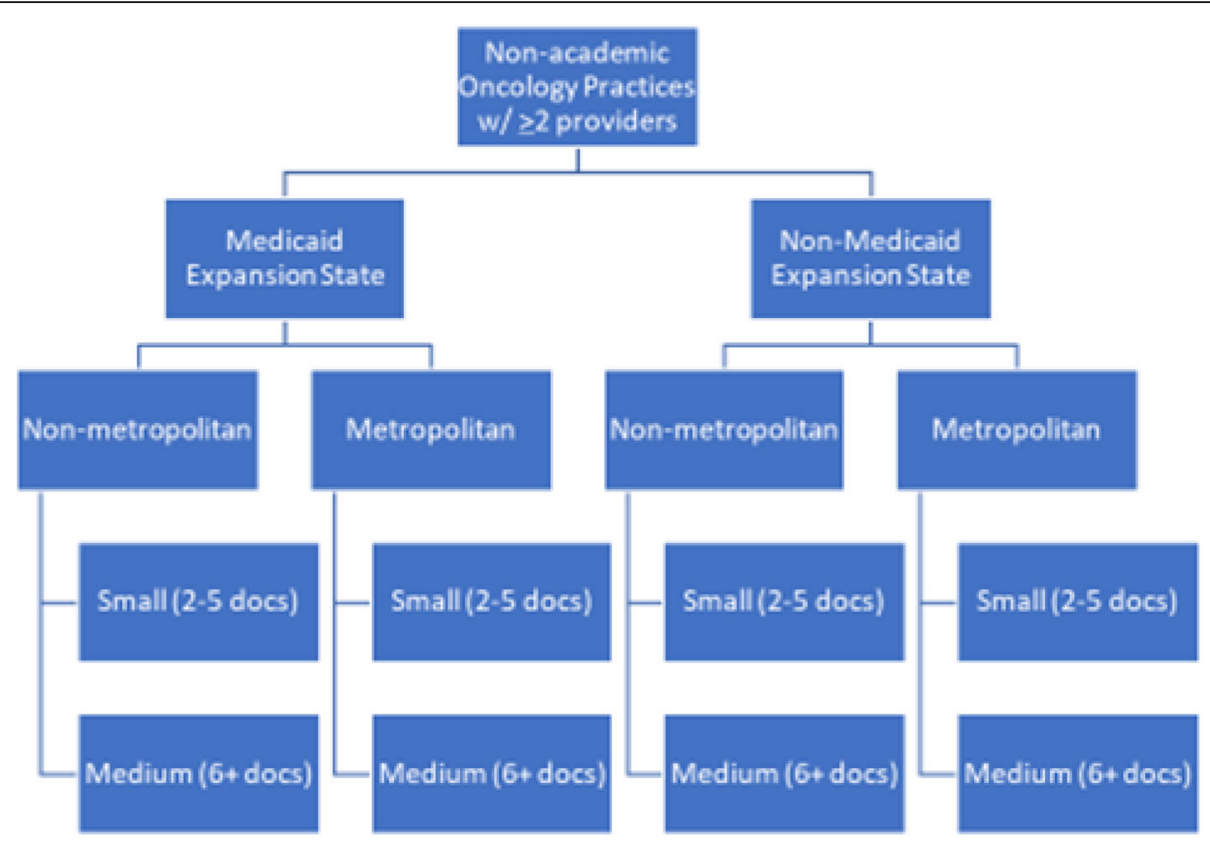

Fig. 1 Site selection to ensure variation in targeted therapy guideline adherence

We determined the sample size of 4121 patients across 8 practices by simulation, accounting for the clustering of patient observations within organizations. We estimated per physician case counts based on national estimates of annual cancer incidence [50], adjusted for stage of cancer appropriate for targeted therapy use [51] and the number of practicing oncologists in the USA [37]. We further estimated the average practice size of small and large community oncology practices based on the American Society of Clinical Oncology's annual census [37] and determined the approximate number of breast, colorectal, lung, and melanoma skin cancer cases which would be potentially eligible for targeted therapy in a 3year study window. This sample size will be adequate to differentiate a modest effect of geographic characteristics on TT use separately in breast, lung, colorectal, and melanoma skin cancer. Given the distributions of cancer incidence, at $80 \%$ power, this sample size will enable the detection of an effect size (absolute difference in rates) of $<0.05$ in breast and lung cancer TTs, an effect size of $<0.10$ in colon cancer, and an effect size of $<0.15$ in melanoma skin cancer TTs for which there are far fewer cases. Effect sizes of these respective magnitudes should represent real differences, within each cancer type. Power simulation, for each cancer type, is shown in Fig. 2.

To assess the determinants of adherence for the qualitative study (aim 2), we will use a snowball sampling technique to identify individuals involved in the process of TT implementation including, but not limited to, physicians, pathologists, pharmacy administrators, cancer center administrators, and genetic counselors. Up to five participants will be interviewed per site. Thus, aim 2 will include $\sim 40$ participants from 8 sites. For qualitative research, sample adequacy is determined by saturation rather than effect size [52]. Based on similar studies [23], we estimate 4 sites of each practice type (high vs low adherers) will be adequate to reach saturation but will monitor the need for additional site recruitment if participant responses are not consistent and saturation is not reached.

\section{Recruitment}

Practices will be recruited using evidence-based approaches to improve response [53] based on social networks of opinion leaders proven successful in our previous studies [54-56]. We will first mail a letter to non-academic practicing oncologists in the catchment area to invite participation and notify them of the participation incentives (\$4000/practice and \$200 gift card/ individual) $[57,58]$. We will also publicize the study among extant networks (e.g., ASCO state organizations, Women in Oncology social media group). Practices in each of the eight aforementioned categories will then be sampled randomly without replacement and approached by email and phone using endorsements from local opinion leaders until we have recruited one practice in each category. Once the practice is enrolled, we will ask the index oncologist to help identify the other key informants within the organization (e.g., physicians, staff, pharmacists, pathologists, administrators) deemed integral to targeted therapy use by the index physician. 
a.

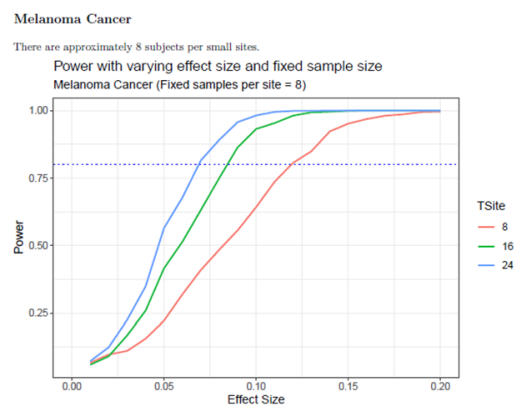

b.

Breast Cancer

There are npproximutely 144 sabjects per small stiter

Power with varying effect size and fixed sample size

Breast Cancer (Fixed samples per site 144)
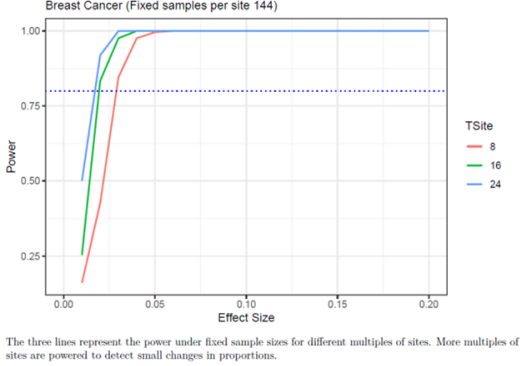

C.

Lung Cancer

There are approximately 65 subjects per small sites

Power with varying effect size and fixed sample size Lung Cancer (Fixed samples

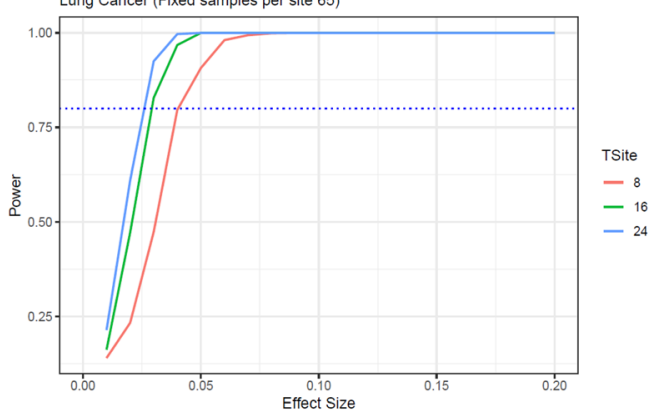

d.

Colon Cancer

There are approximately 21 subjects per small sites.

Power with varying effect size and fixed sample size Colon Cancer (Fixed samples per site 21)

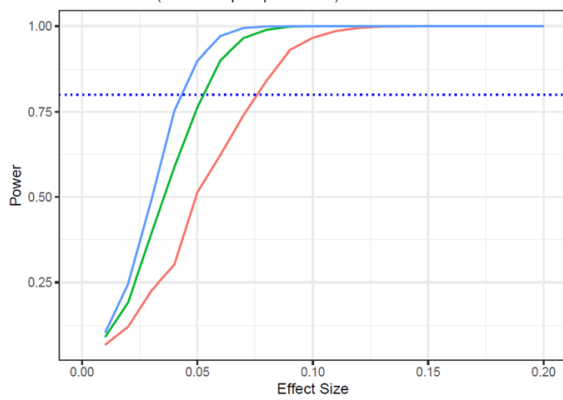

Fig. 2 a-d Power simulation assuming 8, 16, and 24 clusters

\section{Measures}

The dependent variable for aim 1 is appropriate TT use, measured at the patient level and determined by secondary analysis of medical record data. We will assess all eligible treatment opportunities within the study window (January 1, 2016, to July 31, 2019) among patients presenting with their first and only breast, lung, colon, or melanoma tumor at which all or part of the first course of treatment is delivered at a participating site and whose documented care is concordant with 5 breast, 5 lung, 6 colorectal, and 3 melanoma TT measures (Table 1 describes each measure). Each measure is based on the National Comprehensive Cancer Network clinical practice guidelines in effect during the study window. A subset of these measures is validated and maintained by the National Quality Forum (NQF), and new measures are modeled after NQF standards to facilitate future adoption in the field. Each will be assessed for face validity among cancer specialists treating that type of cancer prior to operationalization.

The primary independent variable of interest is patient rurality, measured using HRSA categorizations of rurality mapped to patient zip code recorded in the registry and collected according to institutional registry standards. Other covariates of interest include cancer type (breast, colorectal, lung, melanoma), practice rurality (as measured by HRSA definitions from the practice's clinical office zip code), practice size (small, 2-5 providers, vs large, $\geq 6$ providers), and state public insurance (Medicaid expansion state vs not).

Models will control for patient age, patient insurance type (government-sponsored, private, or none), patient race/ethnicity, patient comorbidity (assessed by the Charlson Comorbidity Index), and clustering of patients within site.

Aim 2 measures are derived from the constructs identified in the Theoretical Domains Framework (TDF). We selected the TDF as our determinant model [25] based on its relevance in previous work to understand the determinants of other cancer specialists' treatment behavior $[22,59]$. The TDF also integrates with the Behavior Change Wheel (BCW) approach to implementation strategy design [60], consistent with our overarching intention to design implementation strategies to support appropriate targeted therapy [61]. We have specified the target behavior across various test-therapy pairs as illustrated in Fig. 3 and specified end users responsible for each step, which prepares us to identify determinants of each step [62, 63]. The TDF consolidates 84 behavioral constructs into a single framework organized into 14 validated domains relevant to health practitioner clinical behavior change [24]. This work will focus on the 14 domains consistent with healthcare provider motivation, capability, and opportunity to adopt and implement targeted therapy. An 
Table 1. Targeted Therapy Measures

\begin{tabular}{|c|c|c|}
\hline Measure & Numerator & Denominator \\
\hline $\begin{array}{l}\text { HER2 testing for overexpression or gene } \\
\text { amplification in patients with breast cancer }\end{array}$ & HER2 testing performed & Adult women with breast cancer \\
\hline $\begin{array}{l}\text { Combination chemotherapy recommended or } \\
\text { administered within } 4 \text { months for women }<70 \\
\text { with AJCC T1cNOMO, or stage IB-III hormone } \\
\text { receptor-negative breast cancer }\end{array}$ & $\begin{array}{l}\text { Combination chemotherapy is } \\
\text { administered within } 120 \text { days of the date } \\
\text { of diagnosis or it is recommended and not } \\
\text { received }\end{array}$ & $\begin{array}{l}\text { Women age } 18-69 \text { diagnosed with stage IB-III } \\
\text { hormone receptor-negative breast cancer (AJCC } \\
\text { T1 CNOMO) }\end{array}$ \\
\hline
\end{tabular}

Adjuvant hormonal therapy recommended or administered within 1 year of diagnosis for women with AJCC t1 CNOMO or stage IB-III hormone receptor-positive breast cancer

Trastuzumab administered to patients with AJCC stage I (T1C)-III and HER2-positive breast cancer who receive adjuvant chemotherapy

HER2-negative or undocumented breast cancer patients spared treatment with HER2-targeted therapies

PDL1 molecular testing of lung cancer patients presenting with advanced or metastatic disease performed within 3 months of diagnosis and prior to systemic therapy

Molecular testing of lung cancer patients presenting with advanced or metastatic disease performed within 3 months of diagnosis

Targeted therapy with a checkpoint inhibitor for lung cancer patients with advanced or metastatic disease and PD-L1 > 1\% and negative test results for EGFR mutations and ALK fusion within 3 months of diagnosis

Targeted therapy with a TKI for lung cancer patients with advanced or metastatic disease and positive test results for EGFR mutations within 3 months of diagnosis

Targeted therapy with ALK inhibitor provided for patients with advanced or metastatic disease and positive test results for ALK fusion within 3 months of diagnosis

RAS (KRAS and NRAS) testing for patients with metastatic colorectal cancer who received anti-EGFR monoclonal antibody therapy

Patients with metastatic colorectal cancer and RAS (KRAS or NRAS) gene mutation spared treatment with anti-EGFR monoclonal antibodies

MMR or MSI testing for patients with metastatic colorectal cancer who receive immune checkpoint inhibitors (pembrolizumab, nivolumab, and ipilimumab)

Patients with metastatic colorectal cancer and MMR or MSI high spared immune checkpoint inhibitors

BRAF testing for patients with metastatic colorectal cancer who receive BRAF inhibitors

Patients with metastatic colorectal cancer and a BRAF gene mutation spared treatment with a BRAF inhibitor

Molecular testing of patients presenting with stage III or stage IV distant metastatic melanoma

Melanoma patients without BRAF V600 mutations or V600 exon 11 or exon 15 mutations spared BRAF inhibitors
Hormone therapy administered within 365 days of the date of diagnosis or it is recommended but not received

Trastuzumab administered within 12 months of diagnosis

HER2 therapies not administered during the initial course of treatment

PD-L1 testing performed prior to systemic therapy date

EGFR and ALK testing performed prior to the systemic therapy start date

Single-agent pembrolizumab delivered within 3 months of diagnosis

Osimertinib, erlotinib, afatinib, gefitinib, or dacomitinib delivered within 3 months of diagnosis

Alectinib, brigatinib, ceritinib, or crizotinib delivered within 3 months of diagnosis

RAS gene mutation testing performed before initiation of anti-EGFR monoclonal antibodies

Anti-EGFR monoclonal antibody therapy not received within the reporting period

MMR or MSI testing performed before initiation of pembrolizumab, nivolumab, and ipilimumab

Immune checkpoint therapy (pembrolizumab, nivolumab, and ipilimumab) not received

BRAF gene mutation testing performed before initiation of BRAF inhibitor

BRAF inhibitor not received

BRAF testing performed

BRAF inhibitor use
Adult women diagnosed with AJCC T1CNOMO or stage IB-III hormone-positive breast tumor

Adult women with AJCC stage I (T1C)-III or HER2positive breast cancer who receive chemotherapy

Adult women with breast cancer that are HER2negative or HER2-undocumented

Adult patients with stage IV NSC lung cancer of histologic subtype adenocarcinoma, large cell, NSCLC NOS, or squamous cell carcinoma

Adult patients with stage IV NSCLC with histologic subtype adenocarcinoma or NSCLC NOS

Adult patients with stage IV NSCLC of histologic subtype adenocarcinoma, NSCLC NOS, or squamous cell carcinoma who are EGFR-negative and ALK-negative

Adult patients with stage IV NSCLC with histologic subtype adenocarcinoma or NSCLC NOS, who are EGFR-positive

Adult patients with stage IV NSCLC of histologic subtype adenocarcinoma, large cell, or NSCLC NOS, who are positive for AKL fusion

Adult patients with metastatic colorectal cancer who receive anti-EGFR monoclonal antibody therapy

Adult patients with metastatic colorectal cancer who have a RAS (KRAS or NRAS) gene mutation

Adult patients with metastatic colorectal cancer who receive pembrolizumab, nivolumab, and ipilimumab

Adult patients with metastatic colorectal cancer who have an MMR or MSI high

Adult patients with metastatic colorectal cancer who receive BRAF inhibitor

Adult patients with metastatic colorectal cancer who have a BRAF mutation

Patients $>18$ presenting with the first and only tumor of stage III or IV melanoma

Patients $>18$ with BRAF-negative, the first and only stage III or IV melanoma 
Table 1. Targeted Therapy Measures (Continued)

\begin{tabular}{lll}
\hline Measure & Numerator & Denominator \\
\hline $\begin{array}{l}\text { Melanoma patients with BRAF V600 mutations } \\
\text { receiving BRAF inhibitors in combination with }\end{array}$ & BRAF inhibitor AND MEK inhibitor use & $\begin{array}{l}\text { Patients } \geq 18 \text { with BRAF-positive, the first and only } \\
\text { stage III or IV melanoma }\end{array}$ \\
MEK inhibitors & & \\
\hline
\end{tabular}

example of the structured interview guide is provided in Appendix, modeled on a previous qualitative assessment of determinants of implementation [64]. The guide elicits information on providers' knowledge and skills in using TTs, TT's alignment with oncologists' social/professional role and identity; beliefs about the capability to engage in TTs; beliefs about consequences of using TTs; motivation and goals for using TTs; memory, attention, and decision processes relevant to TT use; environmental context and resources needed; social influences on TTs; emotion about engaging patients; and ability to regulate TT use.

\section{Data collection}

Most of the data to derive aim 1 measures will be obtained from institutional tumor registries and repositories of information on cancer cases and treatment, collected according to the national standardized data item and coding definitions available from the Commission on Cancer's Facility Oncology Registry Data Standards. Additional testing and treatment data not collected for the registries will be abstracted from medical records. All data will be transferred as a limited data set via REDCap (Research Electronic Data CAPture) [65, 66]. Sites can opt to abstract medical record data using internal resources or allow a study-provided abstractor access.

Aim 2 data will be collected on-site or by phone by trained qualitative interviewers according to the interview guide. Interviews will be recorded and transcribed verbatim. Two pilot interviews will be conducted to ensure consistency in data collection among interviewers.

\section{Data analysis}

For the analysis of data collected in aim 1, we will use descriptive statistics (chi-square, Student's $t$ test, or Fisher's exact test, as appropriate to each variable) to compare individual and organization characteristics of (1) responders and non-responders to recruitment and (2) receipt of targeted therapy among rural and non-rural patients. We will employ hierarchical, multivariable regression modeling to identify the provider and organizational factors associated with TT use, adjusted for patient-level characteristics associated with the use and clustering of patients within the site. We will partition within-site (provider-level differences) and betweensite (organizational-level differences) variance to prioritize aim 2 qualitative comparisons.
Aim 2's qualitative analysis will be a template analysis of TDF domains and subsequent comparative thematic analysis of relevant between-site and within-site differences. A codebook based on TDF constructs was developed a priori. Relevant theoretical domains will be identified through thematic coding of interview transcripts, identification of specific beliefs within coded text units, and mapping of specific beliefs onto theoretical domains [64]. Statements will be further coded as positive (facilitator) or negative (barrier) and by underlying theoretical constructs within TDF domains as necessary. Two team members (SE and JB) will code common interviews to establish interrater reliability. Disagreements will be reconciled by the rest of the study team. The initial eight interview sets (adopters/non-adopters) will be evaluated to identify the range of constructs captured and assessed for saturation. Additional interviews in each practice type will be conducted if thematic saturation is not reached. The relevance of theoretical domains will be assessed by (1) frequency of specific beliefs observed, (2) identification of conflicting beliefs across participants, and (3) evidence of strong beliefs (i.e., participants indicate a specific belief did/would contribute to DA use) [64].

A final, integrated analysis will occur comparing high and low users of TTs. Treatment data from the aim 1 quantitative study will be aggregated at the practice level to rank oncology practices' adherence to targeted cancer therapy guidelines to inform the qualitative analysis. Oncology practices above and below the 50th percentile in the rankings will be designated as high and low users, respectively. Determinants of TT use will be compared between high and low users to isolate determinants most likely salient to TT use and those that most likely limit use. Identified determinants will then be mapped to implementation strategies and delivery modalities suggested in the Behavioral Change Wheel as appropriate approaches using an intervention mapping approach $[67,68]$.

\section{Limitations}

The primary potential challenge to this project is an inability to collect the quantitative data needed for aim 1 in a timely manner. To minimize this risk, we have created data collection protocols which rely on extant data collection at the institutional level for the bulk of the data used. Institutional tumor registries are mandated by law to provide patient characteristics, tumor characteristics, and some molecular testing and results data on an ongoing basis. We have selected measures which can 


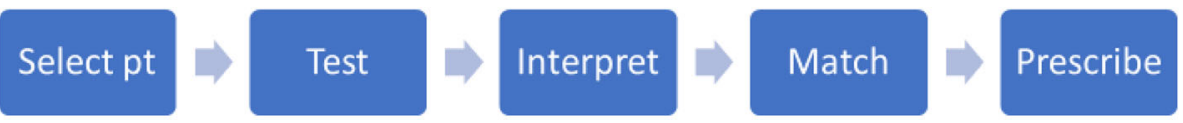

Fig. 3 Target behaviors

leverage these legal requirements for data collection and further selected study windows which coincide with guideline changes and typical data collection backlogs to ensure the data collection is both pragmatic and relevant. We will also make available external contractors to abstract the remaining data elements should participating sites not have capacity. Nonetheless, we will collect the qualitative data first and anticipate that much can be gleaned from determinant analysis relying on physician self-report of TT use if necessary, as we have established in other studies $[23,59]$. Delayed collection could provide opportunities for validation of the two approaches, providing additional methodological contributions of this project. Regardless of the time frame needed for data abstraction completion, our ability to assess the feasibility of this innovative method will advance the field of implementation science. Not only will we provide some of the first estimates of time to transfer of limited data sets, we will also collect critical information regarding the local abstraction processes, electronic infrastructure, and capabilities of community cancer programs which can inform the cost and future approach to test the effectiveness of our implementation strategy.

Secondly, precision medicine adoption rates in the central USA are unknown, so there is potential for adoption rates to be low and few participants to represent adopting organizations. However, we are aware of community practices within our local metropolitan area who advertise precision medicine programs [69] and can identify sites who have participated in both the NCI-MATCH trial and ASCO's TAPUR study. Thus, we can supplement our qualitative sampling frame by directly including such sites, should implementation be low in the sampled sites.

\section{Discussion}

Despite unprecedented investment in precision medicine [70, 71], very little of it has been devoted to translation into practice [72]. Dissemination and implementation research are currently underrepresented in both funding and publications of genomics research. Less than $2 \%$ of funded genomics research is post "bench to bedside," and less than 1\% of published cancer genomic research is post bedside [72]. Consequently, our study will be among the first $[40,73]$ to answer the call to develop a precision delivery initiative to match the precision medicine initiative [74].

Despite the incomplete understanding of determinants driving targeted therapy, several interventions have been launched to accelerate the adoption of genomic medicine [28-36]. Attempts to educate providers through training programs and tumor boards [28-32] have been associated with greater use of testing, but audiences for these efforts are often limited to academic settings. Seventy-nine percent of oncologists practice in non-academic settings [37], typically small, single-specialty practices with far fewer organizational resources available to implement best practices. Other efforts rely on electronic decision support [36]. For example, the IGNITE program's efforts to integrate precision medicine into practice have mostly focused on managing and integrating large amounts of data at the point of care through a single electronic health record (EHR) [36] widely used in academic practice, but far less often used by community practices, who mostly rely on less well-known EHRs [75]. Some rural cancer providers use EHRs which may inadequately support quality cancer care [62]. Consequently, existing efforts to speed adoption of precision medicine may be a poor fit for community practices, particularly those serving rural communities. Implementation strategies designed for community practicing oncologists to fit the practice settings in which they practice, and most cancer patients receive their care, are vital to realizing the full potential of precision medicine.

Once demonstrated to be effective, innovations in care typically take an average of 17 years to reach clinical practice [76]. Implementation science accelerates passive diffusion with evidence-based implementation interventions. However, interventions that are not linked to determinants of the behaviors underlying the care innovations are unlikely to work [25, 68, 77]. Implementation science offers a theoretical framework and methods to specify behaviors and end users, identify determinants (i.e., barriers and facilitators) of end users' behaviors, and more precisely map determinants of behavior to implementation strategies demonstrated to address them [78]. Like targeting tumor characteristics with treatments responsive to them, systematic, empirically based implementation science approaches can target barriers to innovation adoption by matching them with evidence-based implementation strategies demonstrated to increase innovation uptake [68].

We anticipate that barriers to precision medicine implementation identified in aim 1 will be mapped to strategies known to be effective in addressing them, resulting in the development of novel implementation strategies designed for community oncology settings to increase 
innovation-system fit. Identification of implementation strategies could support a cluster-randomized trial of the effectiveness and scalability of precision medicine implementation strategies. Effect sizes and other measurement properties identified in aim 1 can inform sample size estimates for any future trial. Preliminary testing of the measures may establish their face validity and feasibility for future quality improvement efforts. Data collected can be used as baseline rates for a subsequent trial.

Ultimately, this work could potentially expedite society's return on investment in the precision medicine initiative by more rapidly identifying implementation barriers so they can be mapped to effective implementation strategies proven to address them, speeding interventions that can contribute to delays in tumor progression, longer survival, and enhanced quality of life for cancer patients seen across the healthcare delivery system.

\section{Supplementary information}

Supplementary information accompanies this paper at https://doi.org/10. 1186/s43058-020-00064-y.

Additional file 1. StaRI Checklist.

\section{Appendix}

Interview guide: Identifying barriers and facilitators to targeted therapy

\section{Introduction:}

Thank you for agreeing to participate in this interview.

- Is this still a good time for you? Are you in a place where you can be free from distractions and feel free to give candid responses?

- Would it be OK with you if I record this interview for research and training purposes?

We sent you the study information sheet and I would be happy to go over that with you if you have not had a chance to review it. The aim of the study is to help us understand more about the use of targeted cancer therapy. Targeted therapy is the combined use of genetic or other biomarker tests of tumor tissue or specimens and subsequent use of specific drugs or biologics known to produce better outcomes in patients with those specific biomarkers.

Your responses will help us understand how best to implement targeted therapy in practice. All your responses will remain confidential and will only be reported in aggregate. You may choose to stop the interview at any time, and there is no penalty to your or your organization for not completing the interview. If you complete the interview, we will offer you a $\$ 100$ Visa debit card to thank you for your time.

Do you consent to participating in this interview? Do you have any questions before we begin?

\section{The innovation:}

1. I don't have a clinical background, so can you help me understand the role of targeted therapy in cancer treatment?

2. "What do you think about targeted therapy?

3. How does targeted therapy change patient outcomes? Patient care? Your practice? (Beliefs about consequences)

4. "What are (other) benefits of using targeted therapy?

- Prompts: ... to patients, colleagues, cancer program, self: promotion, cancer program accreditation, following guidelines, being respected by colleagues, etc.

5. "What are the main questions or concerns you have about targeted therapy? Are there any costs?

- Prompts: e.g., time taken away from other tasks; effort to keep up; low payoff of finding a positive result

6. Do you that feel the benefits of using targeted therapy outweigh the costs? How so?

7. What alternative therapies could be used instead of targeted therapies? (relative advantage)

- Prompts: What is the advantage of any competing therapeutic approaches?

8. How does using targeted therapy differ from what you did previously to treat cancer patients?

- Prompts: In terms of logistics. What needs to be done differently? Has it changed what you do or what others do? Is this different in breast, lung, colon, melanoma?

\section{Practice:}

9. "What targeted therapy do you offer? (intentions)

- For breast cancer? Colon cancer? Lung cancer? Melanoma?

10. "What genetic test assays do you have access to here? How accessible are they? What genetic tests do you need that you don't have access to? Why?

- Prompts: In-house labs? External? Contractual arrangements with labs? 


\section{Motivation:}

11. Why do you offer those tests and treatments as opposed to others, or for those conditions rather than others?

- Is it required or recommended? By internal or external groups?

12. "What motivated you to adopt targeted therapy or might in cases where you don't currently use it?

13. How important is it to you to use targeted therapy?

- Prompts: How much do you want to do it? Are you compelled to do it? Is there anything more important? Why?

14. "To what extent would you like to see targeted therapy used in your practice in the future? (Goals)

15. How likely is that to happen? (optimism)

16. "Tell me what other influential individuals or groups say about using targeted therapy? (Social influences)

- Prompts: e.g., clinical leaders, management, patients, pharmaceutical representatives, etc.

17. Do you think about the opinions of these influential people when considering whether or how you use targeted therapy?

- Prompts: If you got the sense that others didn't approve of targeted therapy, would that influence whether or how you use targeted therapy?

18. How do clinical practice guidelines support or detract from your use of targeted therapy?

- Prompts: build your confidence/belief in the treatment? Help your efforts to implement targeted therapy across your organization? Which guidelines do you look to for targeted cancer therapy? How do you feel about its quality, clarity, supporting evidence, consistency with other guidelines?

19. Who do you talk to about using targeted therapy? (Social influences)

20. What positive or negative emotions do you experience when using or trying to use targeted therapy? (Emotions)

- Prompts: Do you ever feel any positive emotions? What about negative emotions? Do these emotions differ from when you first started using targeted therapy? Can you imagine how someone new at using targeted therapy might feel? E.g., empathetic, hopeful, positive, resentful, stressed, anxious, content, excited?

21. How do you think your feelings (reference what they said in prior response) influence your use of targeted therapy?

- Prompts: e.g., if you feel anxious about using targeted therapy, are you less likely to use it?

\section{Nature of the behavior:}

"I'd like you to walk me through the steps you take in using targeted therapy. (sketch a process map)

- Prompts for the section: Does it depend? If so, what does it depend on? Which types of cancer? Which characteristics of the cancer (stage, grade)? Do you order the tests or is it automatic? Does the referring provider order it? Do you receive a specific targeted therapy testing result? Does someone ask you to use targeted therapy? Then what happens? When you've ordered a genetic test, do you hand it off to anyone? Is it different for breast, colon, lung, melanoma?

22. First, how do you prepare for using targeted therapy? Is there a method that you use to prepare to use targeted therapy? Is it hard to know when to use targeted therapy?

- Prompts: e.g., read up on recommended cancer tests/treatments? Obtain guideline? Schedule appointment? 
23. How are patients selected to be tested (what methods are used to select them/how do you remember to select them)?

24. How are tests ordered?

25. How are test results reported and interpreted?

26. How are treatments selected to match results (what methods do you use to select treatments/how do you remember to do it/remember the rules)? How are treatments prescribed?

27. What difficulties do you encounter when using targeted therapy?

- Prompts: information technology issues; social conflicts; problems with lab results, drug approvals; pushback from staff or management; lack of reimbursement, support from managers, training, grant funding, reimbursement, staffing, own time; How do the difficulties you encounter when using targeted therapy now differ from the difficulties you encountered when you first started using targeted therapy? What kind of difficulties do you imagine someone new at using targeted therapy might encounter?

28. What procedures or ways of working might encourage you to use targeted therapy?

- Prompts: Audits, reminders, protocol or policy, or monitoring of your behavior (e.g., as part of quality audits)? Feedback on your targeted therapy use? Incentives?

\section{Opportunity}

29. "How often do you see patients who you would assess for targeted therapy?

30. How do you know if your use of targeted therapy is appropriate?

Prompts: Are you aware of the extent to which you are identifying the right patients? Getting the treatments matched to results?

31. In what way is using targeted therapy compatible with other things that you do in your job? Prompts: How? Does using targeted therapy eliminate, minimize, or somehow facilitate your other duties? Or does using targeted therapy make it harder to perform your other duties?

32. "In your opinion, how much does using targeted therapy align with what somebody in your position should be doing?

Prompts: e.g., Is ordering genetic tests something that a nurse/doctor/pathologist should be expected to do? Is matching treatment to genetic test results something that a nurse should be expected to do? Is testing tissue for genetic markers something a pathologist should be doing?

33. Are there ever situations in which you get distracted or forget to use targeted therapy? If so, could you please give me an example?

Prompts: e.g., an urgent patient situation arises; something more important comes up; a colleague interrupts you; thinking about the next client; $6 \mathrm{pm}$ on a Friday and running late to pick up kids

34. How do you know if your use of targeted therapy is appropriate?

Prompts: Are you aware of the extent to which you are identifying the right patients? Getting the treatments matched to results?

35. *We have talked about a lot of things that influence your use of targeted therapy, but what are the main barriers and facilitators to you using targeted therapy as you defined them above?

36. "What strategies have you used to try to overcome these barriers or formalize these facilitators? How did it work? Would you recommend it to others? Prompts: selecting patients to be tested, ordering or recommending the test, interpreting test results, selecting treatment to match results, prescribing treatments

37. "What additional help or support would be most useful for using targeted therapy? Who do you think should provide it?

Prompts: Which patients? Which disease? Which cancer stage?

Capability: Ok, now I would like to ask you some questions about your knowledge and experiences using targeted therapies.

38. What are some of the skills that you use when using targeted therapy?

Prompts: What skills would a healthcare provider just beginning to use targeted therapy need to have or develop to effectively use targeted therapy? If you were to hire someone to do what you do, what sort of skills would they need? e.g., knowledge of genetics, pharmacology, information technology; research; communication 
39. How confident are you in using targeted therapy despite these difficulties?

40. How confident are you that your clinical team can use targeted therapy?

41. "Are there any areas related to targeted therapy where you would like more training or information? Prompt: What additional skills training do you need? What additional skills training do others on your clinical team need?

\section{Conclusion:}

42. "Who else is involved in your delivery of targeted therapy? Would you be willing to introduce me to him/her?

43. "That is all the questions I have for you, has anything occurred to you about this topic that I haven't asked about?

\section{Abbreviations}

Tा: Targeted therapy; TDF: Theoretical Domains Framework; BCW: Behavior Change Wheel; ASCO: American Society of Clinical Oncology; NQF: National Quality Forum

\section{Acknowledgements}

The following individuals contributed their expertise to enable the design of the study: Arda Peterson, Tim Metcalf, and Kerry Barkman, The University of Kansas Health System; Catherine Bruton and Nikki Mulomo, Truman Medical Center, Marlena Barmann; and Larry Corum and Steven Black, Olathe Cancer Care. Sarah Birken commented on a draft of this manuscript and provided insights into the design of the study.

The authors would like to acknowledge the Kansas Institute of Precision Medicine, its Principal Investigator Andrew Godwin, the parent center grant which hosts the project, and an advisory committee of tumor registrars and community oncology providers, and the Masonic Cancer Alliance disease working group, all of whom provided valuable input to increase the feasibility of the study.

Study data were collected and managed using REDCap electronic data capture tools hosted at the University of Kansas. REDCap (Research Electronic Data (apture) is a secure, web-based software platform designed to support data capture for research studies, providing (1) an intuitive interface for validated data capture, (2) audit trails for tracking data manipulation and export procedures, (3) automated export procedures for seamless data downloads to common statistical packages, and (4) procedures for data integration and interoperability with external sources $[65,66]$

\section{Authors' contributions}

$\mathrm{SE}, \mathrm{EE}, \mathrm{KK}, \mathrm{JT}, \mathrm{EWB}, \mathrm{AK}$, and SP contributed to the conceptualization, design, and operationalization of the study. DR contributed to the data analysis for power calculation and sample selection. SE and JB drafted the manuscript. All authors reviewed the manuscript before publication. The authors read and approved the final manuscript.

\section{Funding}

Funding for the study was provided by the National Institutes for General Medical Sciences (P20GM130423).

\section{Availability of data and materials}

Contributing institutions have not agreed to the further sharing of limited data sets they provided. Qualitative data may be available for re-use upon request to the senior author.

\section{Ethics approval and consent to participate}

This study was reviewed and approved by the University of Kanas Medical Center Institutional Review Board. Participants in the qualitative interviews are required to agree to participate, but documentation of consent has been waived. Patients whose medical record data was included without direct patient identifiers in the limited data set used in the quantitative study are not required to provide informed consent.

\section{Consent for publication}

Not applicable

\section{Competing interests}

The contributing authors have no competing interests.

\section{Author details}

${ }^{1}$ Department of Population Health, University of Kansas School of Medicine, 3901 Rainbow Boulevard, Kansas City, KS 66160, USA. ²Rutgers University, 57 US Highway 1, New Brunswick, NJ 08901-8554, USA.

Received: 22 July 2020 Accepted: 31 July 2020

Published online: 31 August 2020

\section{References}

1. Siegel RL, Miller KD, Jemal A. Cancer statistics, 2017. CA: A Cancer Journal for Clinicians. 2017;67(1):7-30.

2. Patel JN. Cancer pharmacogenomics, challenges in implementation, and patient-focused perspectives. Pharmgenomics Pers Med. 2016;9:65-77.

3. Pritchard CC, Mateo J, Walsh MF, De Sarkar N, Abida W, Beltran H, et al. Inherited DNA-repair gene mutations in men with metastatic prostate cancer. N Engl J Med. 2016;375(5):443-53.

4. Stenehjem DD, Bellows BK, Yager KM, Jones J, Kaldate R, Siebert U, et al. Cost-utility of a prognostic test guiding adjuvant chemotherapy decisions in early-stage non-small cell lung cancer. Oncologist. 2016;21(2):196-204.

5. Sparano JA, Gray RJ, Makower DF, Pritchard KI, Albain KS, Hayes DF, et al. Prospective validation of a 21-gene expression assay in breast cancer. New England Journal of Medicine. 2015;373(21):2005-14.

6. Brixner D, Biltaji E, Bress A, Unni S, Ye X, Mamiya T, et al. The effect of pharmacogenetic profiling with a clinical decision support tool on healthcare resource utilization and estimated costs in the elderly exposed to polypharmacy. J Med Econ. 2016;19(3):213-28.

7. Henley SJ, Anderson RN, Thomas CC, Massetti GM, Peaker B, Richardson LC. Invasive cancer incidence, 2004-2013, and deaths, 2006-2015, in nonmetropolitan and metropolitan counties - United States. MMWR Surveill Summ. 2017;66(14):1-13.

8. Unger JM, Moseley A, Symington B, Chavez-MacGregor M, Ramsey SD, Hershman DL. Geographic distribution and survival outcomes for rural cancer patients treated in clinical trials. J Clin Oncol. 2018;36(15).

9. Greenbaum A, Wiggins C, Meisner AL, Rojo M, Kinney AY, Rajput A. KRAS biomarker testing disparities in colorectal cancer patients in New Mexico. Heliyon. 2017:3(11):e00448.

10. Charlton ME, Karlitz JJ, Schlichting JA, Chen WW, Lynch CF. Factors associated with guideline-recommended KRAS testing in colorectal cancer patients: a population-based study. Am J Clin Oncol. 2017;40(5):498-506.

11. Administration USFaD. Pharmacogenomics: overview of the genomics and targeted therapy group Silver Spring, MD: U. S. Food and Drug Adminsitration; 2017 [Available from: https://www.fda.gov/Drugs/ ScienceResearch/ucm572617.htm.

12. Gingras I, Sonnenblick A, de Azambuja E, Paesmans M, Delaloge S, Aftimos $P$, et al. The current use and attitudes towards tumor genome sequencing in breast cancer. Sci Rep. 2016;6:22517.

13. Roberts MC, Weinberger M, Dusetzina SB, Dinan MA, Reeder-Hayes KE, Carey LA, et al. Racial variation in the uptake of oncotype DX testing for early-stage breast cancer. J Clin Oncol. 2016;34(2):130-8.

14. Enewold $L$, Thomas A. Real-world patterns of EGFR testing and treatment with erlotinib for non-small cell lung cancer in the United States. PLoS One. 2016;11(6):e0156728

15. Reeder-Hayes K, Peacock Hinton S, Meng K, Carey LA, Dusetzina SB. Disparities in use of human epidermal growth hormone receptor 2-targeted therapy for early-stage breast cancer. J Clin Oncol. 2016;34(17):2003-9.

16. Gullapalli RR, Desai KV, Santana-Santos L, Kant JA, Becich MJ. Next generation sequencing in clinical medicine: challenges and lessons for pathology and biomedical informatics. Journal of pathology informatics. 2012:3:40. 
17. Johansen Taber KA, Dickinson BD, Wilson M. The promise and challenges of next-generation genome sequencing for clinical care. JAMA Internal Medicine. 2014;174(2):275-80.

18. Lazure P, Marshall JL, Hayes SM, Murray S. Challenges that hinder the translation of clinical advances into practice: results from an international assessment in colorectal cancer. Clin Colorectal Cancer. 2016;15(1):54-66.

19. Collier R. UK seeks to make DNA testing standard in cancer care. CMAJ. 2017;189(30):E1001-E2.

20. Joosten SEP, Retel VP, Coupe VMH, van den Heuvel MM, van Harten WH. Scenario drafting for early technology assessment of next generation sequencing in clinical oncology. BMC Cancer. 2016;16:66.

21. Pant S, Weiner R, Marton MJ. Navigating the rapids: the development of regulated next-generation sequencing-based clinical trial assays and companion diagnostics. Frontiers in oncology. 2014;4:78.

22. Ellis S, Geana M, Griebling T, McWilliams C, Gills J, Stratton K, et al. Development, acceptability, appropriateness and appeal of a cancer clinical trials implementation intervention for rural- and minority-serving urology practices. Trials. 2019;20(1):578.

23. Ellis SD, Geana M, Mackay CB, Moon DJ, Gills J, Zganjar A, et al. Science in the Heartland: exploring determinants of offering cancer clinical trials in rural-serving community urology practices. Urol Oncol. 2019.

24. Phillips CJ, Marshall AP, Chaves NJ, Jankelowitz SK, Lin IB, Loy CT, et al. Experiences of using the Theoretical Domains Framework across diverse clinical environments: a qualitative study. Journal of multidisciplinary healthcare. 2015;8:139-46.

25. Cane J, O'Connor D, Michie S. Validation of the theoretical domains framework for use in behaviour change and implementation research. Implementation Science: IS. 2012;7:37.

26. Messner DA, Al Naber J, Koay P, Cook-Deegan R, Majumder M, Javitt G, et al. Barriers to clinical adoption of next generation sequencing: perspectives of a policy Delphi panel. Applied \& translational genomics. 2016;10:19-24.

27. Io M. Genomics-enabled learning health care systems: gathering and using genomic information to improve patient care and research: workshop summary. Washington, DC: The National Academices Press; 2015. Available from: https://doi.org/10.17226/21707.

28. Knepper TC, Bell GC, Hicks JK, Padron E, Teer JK, Vo TT, et al. Key lessons learned from Moffitt's Molecular Tumor Board: the Clinical Genomics Action Committee experience. Oncologist. 2017;22(2):144-51.

29. Ortiz MV, Kobos R, Walsh M, Slotkin EK, Roberts S, Berger MF, et al. Integrating genomics into clinical pediatric oncology using the molecular tumor board at the Memorial Sloan Kettering Cancer Center. Pediatr Blood Cancer. 2016;63(8):1368-74.

30. Parker BA, Schwaederle M, Scur MD, Boles SG, Helsten T, Subramanian R, et al. Breast cancer experience of the Molecular Tumor Board at the University of California, San Diego Moores Cancer Center. J Oncol Pract. 2015;11(6):442-9.

31. Schwaederle M, Parker BA, Schwab RB, Fanta PT, Boles SG, Daniels GA, et al. Molecular tumor board: the University of California-San Diego Moores Cancer Center experience. Oncologist. 2014;19(6):631-6.

32. Tafe $L$, Gorlov IP, de Abreu FB, Lefferts JA, Liu X, Pettus JR, et al. Implementation of a molecular tumor board: the impact on treatment decisions for 35 patients evaluated at Dartmouth-Hitchcock Medical Center. Oncologist. 2015;20(9):1011-8.

33. National Human Genome Research Institute. IGNITE: Implementing Genomics in Practice (IGNITE) II: Pragmatic Clinical Trials Network Bethesda, MD: Department of Health and Human Services; 2019 [Available from: https://www.genome.gov/Funded-Programs-Projects/Implementing Genomics-in-Practice-IGNITE-2-Pragmatic-Clinical-Trials-Network.

34. Orlando LA, Sperber NR, Voils C, Nichols M, Myers RA, Wu RR, et al. Developing a common framework for evaluating the implementation of genomic medicine interventions in clinical care: the IGNITE Network's Common Measures Working Group. In: Genet Med; 2017.

35. Sperber NR, Carpenter JS, Cavallari LH, JD L, Cooper-DeHoff RM, Denny JC, et al. Challenges and strategies for implementing genomic services in diverse settings: experiences from the Implementing GeNomics In pracTicE (IGNITE) network. BMC Med Genomics. 2017;10(1):35.

36. Weitzel KW, Alexander M, Bernhardt BA, Calman N, Carey DJ, Cavallari LH, et al. The IGNITE network: a model for genomic medicine implementation and research. BMC Med Genomics. 2016;9:1.

37. Kirkwood MK, Hanley A, Bruinooge SS, Garrett-Mayer E, Levit LA, Schenkel C, et al. The state of oncology practice in America, 2018: Results of the ASCO Practice Census Survey. J Oncol Pract. 2018;14(7):e412-e20.
38. Ellis SD, Geana M, Mackay C, Gills J, Griebling T, Zganjar A, et al. When an intervention is not designed for dissemination: developing a strategy to improve system-innovation fit. 10th Annual Conference on the Science of Dissemination and Implementation; December 4-6, 2017; Arlington, VA2017.

39. Manolio TA. Implementing genomics and pharmacogenomics in the clinic: the National Human Genome Research Institute's genomic medicine portfolio. Atherosclerosis. 2016;253:225-36.

40. Roberts MC, Kennedy AE, Chambers DA, Khoury MJ. The current state of implementation science in genomic medicine: opportunities for improvement. Genet Med. 2017;19(8):858-63.

41. Fetters MD, Curry LA, Creswell JW. Achieving integration in mixed methods designs-principles and practices. Health Serv Res. 2013;48(6 Pt 2):2134-56.

42. Pinnock H, Barwick M, Carpenter CR, Eldridge S, Grandes G, Griffiths CJ, et al. Standards for Reporting Implementation Studies (StaRI) Statement. BMJ. 2017;356:16795.

43. Pinnock H, Epiphaniou E, Sheikh A, Griffiths C, Eldridge S, Craig P, et al. Developing standards for reporting implementation studies of complex interventions (StaRI): a systematic review and e-Delphi. Implement Sci. 2015; 10:42.

44. O'Brien BC, Harris IB, Beckman TJ, Reed DA, Cook DA. Standards for reporting qualitative research: a synthesis of recommendations. Acad Med. 2014;89(9):1245-51.

45. United States Census Bureau. Geographic areas and rural data: nation and region suitland, MD: United States Census Bureau; 2020 [Available from: https://gis-portal.data.census.gov/arcgis/apps/MapSeries/index.html?appid= 7a41374f6b03456e9d138cb014711e01.

46. Weaver KE, Geiger AM, Lu L, Case LD. Rural-urban disparities in health status among US cancer survivors. Cancer. 2013;119(5):1050-7.

47. Blake KD, Moss JL, Gaysynsky A, Srinivasan S, Croyle RT. Making the case for investment in rural cancer control: an analysis of rural cancer incidence, mortality, and funding trends. Cancer Epidemiol Biomarkers Prev. 2017;26(7): 992-7.

48. Muthukrishnan M, Sutcliffe S, Hunleth JM, Wang JS, Colditz GA, James AS. Conducting a randomized trial in rural and urban safety-net health centers: added value of community-based participatory research. Contemp Clin Trials Commun. 2018;10:29-35.

49. Gorospe JR. Introducing the Institutional Development Award (IDeA) Program Bethesda. U.S. Department of Health and Human Services: MD; 2012

50. Group USCSW. U.S. Cancer Statistics Data Visualizations tool based on November 218 submission data (1999-2016) Atlanta, GA2019 [Available from: www.cdc.gov/cancer/dataviz.

51. National Cancer Database (NCDB) [Internet]. American College of Surgeons. 2018.

52. Vasileiou K, Barnett J, Thorpe S, Young T. Characterising and justifying sample size sufficiency in interview-based studies: systematic analysis of qualitative health research over a 15-year period. BMC Med Res Methodol. 2018;18(1):148.

53. Dillman DA, Smyth JD, Christian LM. Internet, phone, mail, and mixed-mode surveys: the tailored design method. 4th ed. Hoboken, NJ: Wiley; 2014.

54. Ellis SD, Bertoni AG, Bonds DE, Clinch CR, Balasubramanyam A, Blackwell C, et al. Value of recruitment strategies used in a primary care practice-based trial. Contemp Clin Trials. 2007;28(3):258-67.

55. Ellis SD, Thrasher JB, Jones EV, Kimminau KS. Treatment decision making in low-risk prostate cancer: recruitment and data collection feasibility. In: Sociey for Medical Decision Making Annual Meeting; October, vol. 2015. St. Louis, MO: Society for Annual Decision Making; 2015.

56. Inc. P, The American Academy of Family Physicians National Research Network. Shared accountability in family medicine: physician and patient perspectives. Princeton, NJ: Robert Wood Johnson Foundation; 2017.

57. Singer $E_{1}$ Couper MP. Do incentives exert undue influence on survey participation? Experimental evidence. J Empir Res Hum Res Ethics. 2008;3(3): 49-56.

58. Dillman DA. Mail and Internet surveys. 2nd Edition, 2007 Edition ed. Hoboken, New Jersey: John Wiley; 2007.

59. Ellis SD, Geana M, Mackay CB, Moon DJ, Gills J, Zganjar A, et al. Science in the Heartland: exploring determinants of offering cancer clinical trials in rural-serving community urology practices. Urol Oncol. 2019;37(8):529 e9e18.

60. Michie S, Atkins L, West R. The Behaviour Change Wheel: a guide to designing interventions. Great Britain: Silverback Publishing; 2014. 
61. Sales A. Implementation science: how can it support health care research? Training in Implementation: Actionable Research Approaches (TIARA); June 19, 2019; Kansas City, KS2019.

62. Zganjar A, Mackay C, Petty L, Geana M, Gills J, Griebling T, et al. LEARNINFORM-RECRUIT: increasing the offer of urologic cancer trials in community practice. South Central Section of the American Urological Association; October 4-7, 2017; Naples, FL2017.

63. Ellis SD, Kimminau K, Jones EV, Petty L, Ellerbeck E, Thrasher JB. Potential barriers to use of prostate cancer treatment decision aids in urology practice. AcademyHealth; June 26-28, 2016; Boston, MA2016.

64. Birken SA, Presseau J, Ellis SD, Gerstel AA, Mayer DK. Potential determinants of health-care professionals' use of survivorship care plans: a qualitative study using the theoretical domains framework. Implementation science: IS. 2014;9(1):167.

65. Harris PA, Taylor R, Minor BL, Elliott V, Fernandez M, O'Neal L, et al. The REDCap Consortium: building an international community of software platform partners. J Biomed Inform. 2019;95:103208.

66. Harris PA, Taylor R, Thielke R, Payne J, Gonzalez N, Conde JG. Research electronic data capture (REDCap)--a metadata-driven methodology and workflow process for providing translational research informatics support. J Biomed Inform. 2009;42(2):377-81.

67. Peskin MF, Hernandez BF, Gabay EK, Cuccaro P, Li DH, Ratliff E, et al. Using intervention mapping for program design and production of iCHAMPSS: an online decision support system to increase adoption, implementation, and maintenance of evidence-based sexual health programs. Front Public Health. 2017:5:203.

68. Rogal SS, Yakovchenko V, Waltz TJ, Powell BJ, Kirchner JE, Proctor EK, et al. The association between implementation strategy use and the uptake of hepatitis $C$ treatment in a national sample. Implementation Science: IS. 2017;12(1):60.

69. System SLsH. Saint Luke's Center for precision oncology Kansas City. In: MO: Saint Luke's Health System; 2017.

70. Byrnes N. Slow progress to better medicine: thirteen years after the human genome was sequenced, some remarkable treatments are being developed. MIT Technology Review. 2016 July;25:2016.

71. Global Market Insights I. GLOBE NEWSWIRE [Internet]. Ocean View, DE2017. [cited 2017].

72. Khoury MJ, Gwinn ML, Glasgow RE, Kramer BS. A population approach to precision medicine. Am J Prev Med. 2012:42(6):639-45.

73. Chambers DA, Feero WG, Khoury MJ. Convergence of implementation science, precision medicine, and the learning health care system: a new model for biomedical research. JAMA. 2016;315(18):1941-2.

74. Parikh RB, Schwartz JS, Navathe AS. Beyond genes and molecules - a precision delivery initiative for precision medicine. N Engl J Med. 2017; 376(17):1609-12

75. Kate M. Top 10 ambulatory EHR vendors by physician practice EHR Implementations. EHR Intelligencecom. 2017.

76. Balas EA, Boren SA. Managing clinical knowledge for health care improvement. Yearb Med Inform. 2000;(1):65-70.

77. Powell BJ, Waltz TJ, Chinman MJ, Damschroder L, Smith JL, Matthieu MM, et al. A refined compilation of implementation strategies: results from the Expert Recommendations for Implementing Change (ERIC) project. Implement Sci. 2015;10:21.

78. Proctor E, Silmere H, Raghavan R, Hovmand P, Aarons G, Bunger A. Outcomes for implementation research: conceptual distinctions, measurement challenges, and research agenda. Admin Pol Ment Health. 2011;38

\section{Publisher's Note}

Springer Nature remains neutral with regard to jurisdictional claims in published maps and institutional affiliations.

Ready to submit your research? Choose BMC and benefit from:

- fast, convenient online submission

- thorough peer review by experienced researchers in your field

- rapid publication on acceptance

- support for research data, including large and complex data types

- gold Open Access which fosters wider collaboration and increased citations

- maximum visibility for your research: over $100 \mathrm{M}$ website views per year

At BMC, research is always in progress.

Learn more biomedcentral.com/submissions 